\title{
Absence of clinical cerebellar syndrome after serial injections of more than 20 doses of gadoterate, a macrocyclic GBCA: a monocenter retrospective study
}

\author{
Gaetano Perrotta $^{1} \cdot$ Thierry Metens $^{2} \cdot$ Julie Absil $^{2} \cdot$ Marc Lemort $^{3} \cdot$ Mario Manto $^{4}$
}

Received: 14 July 2017 / Accepted: 25 September 2017 / Published online: 27 September 2017

(C) Springer-Verlag GmbH Germany 2017

\begin{abstract}
Sound evidence of gadolinium accumulation in brain has been recently provided after repeated administrations of linear gadolinium-based contrast agents (GBCAs), especially at the cerebellum level. Although data regarding brain accumulation of macrocyclic GBCAs are more reassuring, there is now a genuine concern ("gadoliniumphobia") about possible long-term consequences of gadolinium deposits, especially in terms of cerebellar sequelae. $\mathrm{We}$, therefore, questioned about the clinical impact of serial administration of gadoterate meglumine, a macrocyclic GBCA. In this retrospective study (2000-2016) of medical files of patients who received more than 20 administrations of gadoterate, we searched for cerebellar symptoms and signs developing during the regular follow-up. We reviewed medical files of ten patients (mean age $34.4 \pm 20.8$ years; 4 males, 6 females) who received $28.2 \pm 5.3$ doses of gadoterate (average total dose of GBCA $518 \pm 226 \mathrm{ml}$; range 185 $785 \mathrm{ml}$ ). Patients were examined by at least two medical specialists depending on initial diagnosis, and at least once by a neurosurgeon. Mean follow-up time was 91 months (range 49-168) and six out of ten patients experienced new
\end{abstract}

Electronic supplementary material The online version of this article (doi:10.1007/s00415-017-8631-8) contains supplementary material, which is available to authorized users.

Mario Manto

mmanto@ulb.ac.be

1 Service de Neurologie, ULB-Hôpital Erasme, Route de Lennik, Brussels, Belgium

2 Service de Neuroimagerie, ULB-Hôpital Erasme, Route de Lennik, Brussels, Belgium

3 Service de Radiologie, Institut Bordet, Brussels, Belgium

4 FNRS, ULB-Hôpital Erasme, Route de Lennik 808, 1070 Brussels, Belgium symptoms or signs. No clinician reported the appearance of a rising cerebellar syndrome, nor newly appeared symptoms or signs suggested cerebellar toxicity. This retrospective clinical study shows no de novo clinical cerebellar syndrome following repeated administrations of gadoterate. Our results argue against a cerebellar toxicity of this macrocyclic agent. Still, confirmation in a larger number of subjects is required, as well as clinical studies concerning linear GBCAs whose structure and in vivo stability are distinct.

Keywords Gadolinium deposition · Macrocyclic · Linear · Brain deposits · Basal ganglia $\cdot$ Toxic cerebellar syndrome

\section{Introduction}

Recent reports have highlighted that repeated administration of gadolinium-based contrast agents (GBCAs) is associated with an increased signal intensity (SI) in the dentate nucleus (DN) and globus pallidus (GP) on unenhanced T1-weighted magnetic resonance (MR) images [4, 13, 22]. In particular, the dentate nucleus-to-pons SI ratio is significantly correlated with the number of previous GBCAs administrations. The same observation has been made for the globus pallidusto-thalamus SI ratio. Such deposits are not only reported in adults but also in children undergoing multiple MR examinations [10]. It is estimated that more than 10 million intravenous doses of GBCAs are administered annually in the USA [33] and, therefore, accumulation of gadolinium deposits in the brain becomes a hot topic at a world level for the medical community.

Histological evidence of deposits in post-mortem specimen has been provided [19]. Nevertheless, the mechanism of the accumulation of gadolinium in the human brain, 
especially in cerebellar nuclei and basal ganglia, and the long-term fate of these deposits are currently unknown, both from a radiological and from a neurological point of view.

GBCAs are chelated compounds where the relatively toxic $\mathrm{Gd}^{3+}$ ion is complexed in carrier molecules (aminopolycarboxylic acid ligands) to prevent dissociation in the body. GBCAs are unique among pharmaceuticals, being small water soluble entities with paramagnetic properties which shorten the $\mathrm{T} 1$ relaxation time of tissue water [7]. These complexes were previously considered as highly stable [28]; however, after the first reports of nephrogenic systemic fibrosis (NSF) [6] this notion has been challenged and concern is now rising again with the discovery of brain deposits $[13,24]$.

A link between a possible toxicity of GBCAs and renal failure was initially raised with the occurrence of NSF [9]. However, the accumulation of gadolinium in brain structures has been described even without severe renal dysfunction [12] and there is now a real fear about the in vivo stability of GBCAs in subjects with unaltered renal function [19].

Although current studies have focused in particular on the accumulation of gadolinium in dentate nuclei, there is a possibility that gadolinium is also found at higher concentrations in the cerebellar cortex for several reasons: (a) the existence of anatomical nucleo-cortical loops in the cerebellum, (b) a direct neurotoxic effect on cerebellar cortex has been observed following the intra-ventricular administration in animals [25], and (c) latest experimental studies support the notion that cerebellar cortex is a site of deposits [15]. The accumulation in cerebellar cortex is particularly relevant given that, amongst all the cerebellar cells, the Purkinje neurons are especially susceptible to intoxication and poisoning [16].

While there is clear evidence that linear GBCAs are associated with brain accumulation $[4,14]$, much less is known about macrocyclic compounds. Indeed, only one study reported increased signal intensity in dentate nucleus and globus pallidus after repeated administration of gadobutrol, a macrocyclic GBCA [31]. This study has been criticized because no difference of SI was evident in the published figures [1]. Retrospective studies showed no SI increase even after over 20 doses of macrocyclic compounds [21], although autopsy materials provide evidence of $\mathrm{Gd}^{3+}$ accumulation in different brain structures after administration of more stable GBCAs [20].

At this stage, it appears critical to establish whether repeated administration of GBCAs are associated or not with clinically relevant consequences. In this retrospective study, we focused on patients who received serial administrations of the macrocyclic agent gadoterate dimeglumine. We specifically looked for the onset of new neurological symptoms reported by clinicians in medical files. We wondered whether a toxic-induced cerebellar syndrome [16] could be generated by potential gadolinium accumulation.

\section{Methods}

After approval by the local ethics committee (Comité d'Ethique Hospitalo-Facultaire, Hôpital Erasme-ULB, reference P2016/347), we identified in our database patients who received at least four administrations of GBCAs from January 1st, 2000 to December 31st, 2015. From this group, we extracted the list of patients who received exclusively more than 20 administrations of gadoterate meglumine (Dotarem ${ }^{\circledR}$ ) during the same period. The major endpoint was to look for a possible report of a de novo cerebellar syndrome that might have occurred unexpectedly. A comprehensive list of signs and symptoms related to a cerebellar impairment was first established (see Tables 1 for details). Subsequently, accessible medical files were reviewed in depth to collect data concerning the principal diagnosis, birth date, gender, successive dates of MR scans, doses of GBCA, renal function (the estimated glomerular filtration rate, eGFR, at the end of follow-up was computed according to Cockroft [5] or Schwarz [29] depending on the age of the subject), and every newly occurring cerebellar-related sign or symptom as reported by the treating physicians.

As a minor and secondary endpoint, we also attempted to assess brain MR images (results are shown in the Supplementary File). Sequences of acquisition varied largely between patients. For the brain MR analysis, images of first and last available MR scan with an unenhanced 3D T1-weighted sequence (gradient echo; repetition time $9 \mathrm{~ms}$; echo time $4 \mathrm{~ms}$ ) were analyzed to compare SI in the brain regions that have been described to be involved in gadolinium accumulation [14]. Post-processing was performed with Intellispace Portal, (Philips Medical System, The Netherlands). Axial reconstruction was obtained with slice thickness of $3 \mathrm{~mm}$. Regions of interest (ROIs) were placed on right and left $\mathrm{DN}$, frontal white matter (WM), right and left thalamus, DN, central pons, and middle cerebellar peduncle (MCP). The average SI of the right and the left DN was calculated and divided by the mean SI of the ROI of the central pons, to compute the DN-to-pons ratio. We also computed the DN-to-CSF ratio by considering the SI of a ROI located in the 4th ventricle. The average SI of the right and left GP and thalamus was calculated and the GP-to-thalamus ratio was also determined.

\section{Results}

Of 2978 patients who received at least 4 injections of GBCAs, 11 with exclusive administration of gadoterate 
Table 1 List of the cerebellar (a) symptoms that were specifically searched in medical files and (b) signs that were searched in medical files

\begin{tabular}{ll}
\hline (a) Cerebellar symptoms & \\
\hline Headache & 4 \\
Dizziness & 1 \\
Diplopia & 3 \\
Oscillopsia/blurred vision & 0 \\
Speech difficulties & 1 \\
Lack of coordination in limbs & 1 \\
Clumsiness in limbs & 0 \\
Tremor in limbs & 0 \\
Writing difficulties & 0 \\
Gait difficulties & 1 \\
Unsteadiness while standing & 0 \\
Falls & 1 \\
\hline
\end{tabular}

(b) Cerebellar signs

Fixation deficits

Ocular misalignment

Disorder of pursuit

Deficits of saccades

Nystagmus

Abnormal vestibulo-ocular reflex

Abnormal optokinetic response

Dysarthria

Palatal tremor

Dysmetria

Tremor

Hypotonia

Extensor hypertonia; myoclonus

Asynergia

Adiodochokinesia

Dysrhythmokinesia

Abnormal Stewart-Holmes maneuver

Isometrataxia

Writing impairment

Ataxia of stance

Ataxic gait

Attention deficit

Emotion disorders

Aggressivity, irritability

Passivity

Psychosis

Autism spectrum

Right column shows number of occurrences

(more than 20 doses) were extracted from the database. Medical file of one patient was not accessible for research purposes (refusal from the patient) and was thus excluded from the analysis. The relevant data from medical files of the remaining ten subjects are summarized in Table 2 .
Timing of MR scans and onset of new symptoms and signs are shown in Fig. 1.

In terms of main diagnosis, a primary brain tumor was the reason of first consultation for four out of ten patients. The six remaining patients had brain metastasis at the onset or developed brain metastases during the follow-up period. The localization of metastasis is described in Table 2. Five patients died, one (Patient 5) moved abroad; the remaining four patients are continuing the follow-up at the out-patient clinic.

Patients were all followed by at least two specialists depending on diagnosis, including pediatricians, neuropediatricians, dermatologists, oncologists, pneumologists, gynecologists, and neurosurgeons. Of note, each patient was examined at least once by a neurosurgeon. Renal function was normal for all the patients during the observation period. Values of eGFR at the end of follow-up are given in Table 2.

During a mean of $28.2 \pm 5.3$ MR exams per patient, an average dose of $23.3 \pm 9.7 \mathrm{ml}$ of gadoterate per scan was administered (average total dose of contrast agent per patient: $517.7 \pm 226.2 \mathrm{ml}$ ). The average interval between injected MR scans was 85 days. Review of medical files of Patient 9 showed that gadobenate dimeglumine (Multihance $^{\circledR}$ ) was administered three times between MR scan 1 and 5. These MR scans were performed before the period used for database research and, for the following 31 MR scans, only gadoterate meglumine was used. For Patient 1 and Patient 10, records of an external MR scan realized before the beginning of the follow-up were found. We cannot exclude that other MR scans were performed in external centers during the follow-up period. However, no documentation was found in the medical files.

During a mean follow-up time of 91 months (range 49-168), six out of ten patients experienced new signs or symptoms inventoried in Tables 1. Most of them were associated with the main neurological or neurosurgical disorder (see Table 3).

Headache and diplopia were described in Patient 1. This patient had a brain astrocytoma. He developed intracranial hypertension and 6th nerve palsy. Dizziness was described in Patient 7 with multiple brain metastasis (sites: right temporal, right fronto-temporal, frontal interhemispheric, left occipito-parietal and right cerebellar) of a melanoma. Diplopia and limb ataxia were observed in Patient 6 after demonstration of tectal plate metastasis of lung cancer. Speech difficulties in Patient 5 were associated with a left temporal metastasis of melanoma. As for neuropsychological and behavioral symptoms experienced by Patients 1 , 3 , and 10, they were all explained by the primary lesion.

In no medical file a report of an unexpected cerebellar syndrome was found. In particular, no patient showed evidence of a de novo cerebellar motor syndrome (CMS), vestibulo-cerebellar syndrome (VCS) or Schmahmann's 


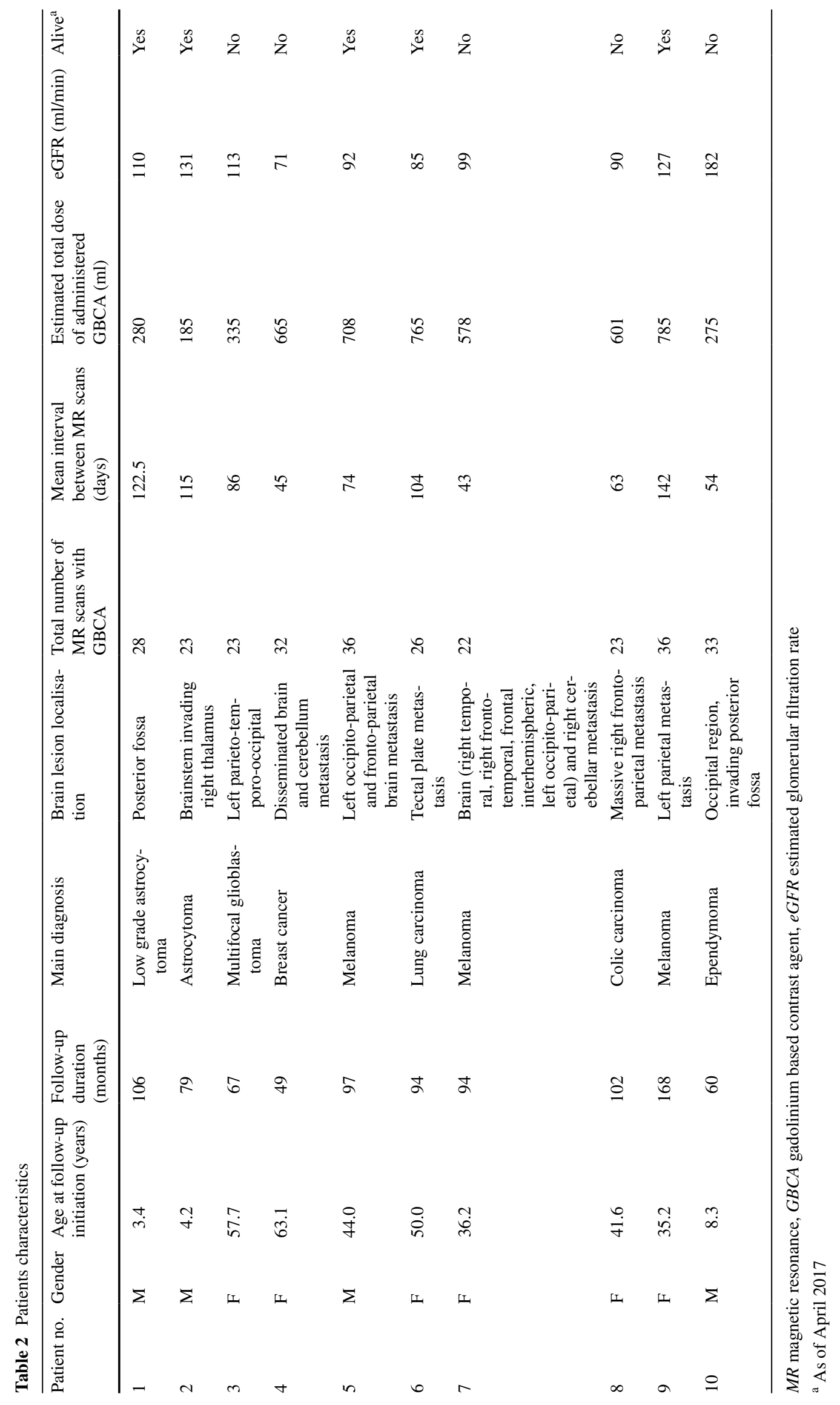




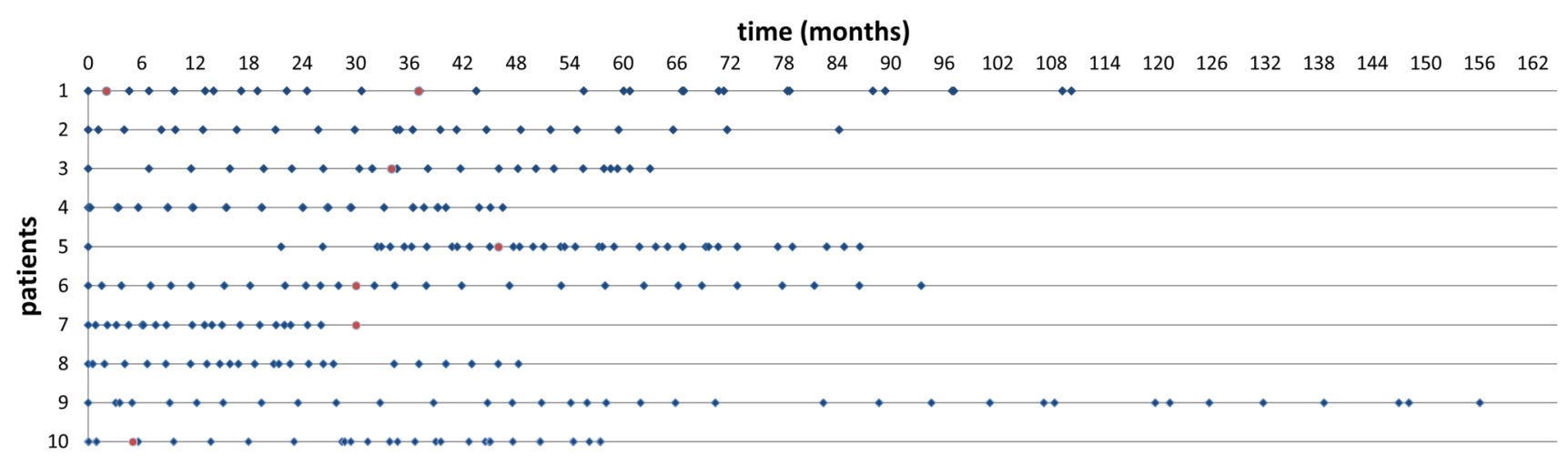

Fig. 1 Temporal distribution of MR scans and newly appeared symptoms/signs during follow-up, as described in the medical files. Each horizontal line corresponds to a patient. Blue diamonds represent MR scans; red dots represent new signs/symptoms. Time is expressed in months

syndrome [17]. No patient developed a cerebellar syndrome as observed after environmental chronic intoxication, in particular after chronic exposure to metals (such as for mercury, lead, manganese, aluminium, thallium, germanium, uranium or vanadium) [16]. No case was suggestive of a subacute encephalopathy involving dentate nuclei as observed after metronidazole administration [8, 32]. Furthermore, we did not observe dose-related symptoms as commonly reported for drugs-induced ataxia [16].

In term of MR image analysis (see Supplementary File), we found MR exams with unenhanced 3D-T1 weighted sequences for six patients. For the only three patients with more than one exam available (Supplementary Fig. 2-4), the SI of ROIs were studied. No SI increase was identified in globi pallidi (GP) nor dentate nuclei (DN) at visual inspection. SI ratios were calculated for GP over Thalamus for the three patients and for DN over pons for only two patients (Patient 2 and Patient 3) because of posterior fossa lesions found in Patient 1 (see Supplementary Table 4). Other calculated SI ratios did not provide additional information (DNto-MCP, GP-to-WM; data not shown). No signal increase was identified on the available images for Patient 8 (7th MR scan, Supplementary Fig. 5) and Patient 9 (15th MR scan, Supplementary Fig. 6). For Patient 10, images of the 32nd scan are shown in Supplementary Fig. 7. No evidence of increase in SI in the basal ganglia nor in the cerebellum could be demonstrated (provided that cerebellar lesion could have hampered the image analysis).

\section{Discussion}

To our knowledge, this is the first retrospective study investigating specifically the clinical consequences of repeated administration of a macrocyclic GBCAs in humans.

We reviewed the medical files of ten patients who received a mean administration of 28 serial injections of
Table 3 Symptoms and signs reported by clinicians in the medical files during the follow-up period

\begin{tabular}{lll}
\hline Symptoms and signs $^{\mathrm{a}}$ & $\begin{array}{l}\text { Number of occur- } \\
\text { rences }\end{array}$ & Patient \\
\hline Headache & 1 & 1 \\
Dizziness & 1 & 7 \\
Diplopia & 2 & $1 ; 6$ \\
Speech difficulties & 1 & 5 \\
Lack of coordination in limbs & 1 & 6 \\
Attention deficit & 3 & $1 ; 3 ; 10$ \\
Aggressiveness, irritability & 1 & 1 \\
Passivity & 1 & 10 \\
\hline
\end{tabular}

${ }^{\text {a }}$ In none of the patients, a genuine toxic-induced cerebellar syndrome was reported in the medical files. Symptoms and signs which could have been suggestive of a cerebellar dysfunction can be explained by the primary tumor or the metastases in all the cases

GBCA. The 20 administrations cut-off were chosen based on preclinical studies showing accumulation of gadolinium in the brain of healthy rats after 20 injections of gadoterate meglumine [15, 26]. Although Radbruch et al. recently confirmed that no signal intensity augmentation can be observed on MR scans after 23 repeated administrations of gadoterate meglumine [21], autopsies have revealed that gadolinium can still accumulate without MR evidence [27]. Therefore, this opens the question of possible clinical consequences for neurologists,/neurosurgeons and even for psychiatrists, given the key-role of the cerebellum in motor/cognitive/affective skills [17].

From the clinical standpoint, the effects of gadolinium accumulation on human brain functions and especially cerebellar functions are nearly completely unknown. Two cases of presumed gadolinium-induced encephalopathy have been described [11, 18]; several publications report a gadolinium deposition disease with several aspecific neurological 
symptoms including headaches, altered mentation, diffuse pain and paresthesias $[3,23,30]$. The link with a genuine cerebellar toxic disorder cannot be made on the basis of these reports. An impaired glucose uptake has been reported in deep brain nuclei and dentate nuclei after GBCA administration [2] but the clinical relevance is unknown.

In our study, on the basis of the current nosology of clinical cerebellar syndromes [17] we did not find any evidence of newly appearing cerebellar symptoms or signs which would fit with an evolving gadolinium-related cerebellar disease. Doubts could be raised about possible new neuropsychological and behavioral symptoms, but there was no temporal association with GBCA administration which would argue for a cerebellar cognitive affective syndrome. None of the patients developed the constellation of symptoms observed in Schmahmann's syndrome [17].

This study has several important limitations. First, due to retrospective nature of the study, clinical information about patients was not reported uniformly. However, it can be argued that each patient was examined by at least two different specialists and medical files were reviewed systematically on the basis of an exhaustive list of symptoms and signs observed in classical cerebellar disorders. Second, all of the ten patients presented initially or developed subsequently intracranial neoplastic lesions. This impacts on the interpretation of novel symptoms, as a result of anatomical disruption of central pathways and raised intra-cranial pressure. Third, MR analysis could not be performed properly: (a) sequences of acquisition varied largely from one patient to another or for successive MR scans in the same patient, (b) 3D-T1 weighted sequences were only available for six patients, (c) a comparison of successive scans was only possible for three patients, (d) intracranial lesions interfered with the analysis of signal intensity of dentate nucleus in Patients 1 and 10 and of right thalamus in Patient 2. Moreover, because of their principal diagnosis, patients underwent chemotherapy, radiotherapy, gamma-knife and/or surgical procedures which could have interfered with SI intensity interpretation. Nevertheless, visual inspection of the interpretable MR scans did not show any SI augmentation similar to those reported previously with linear GBCAs [21].

In conclusion, the analysis of the medical files of 10 patients who received more than 20 repeated administration of gadoterate meglumine did not show any de novo cerebellar syndrome. A larger clinical retrospective study is mandatory. Prospective studies investigating extensively the cerebellar functions of these patients should now be conducted. The same comment applies to linear GBCAs, especially given the recent report of high $\mathrm{Gd}^{3+}$ concentrations not only in the dentate nucleus but also in the granular layer the cerebellar cortex following repeated administrations of gadodiamide and gadopentetate dimeglumine [15]. The combined accumulation in these two key structures of the cerebellum is a matter of concern and requires urgent clinical reports as well as detailed prospective follow-up studies in patients who have been exposed to repeated administrations.

Acknowledgements GP is Ph.D. student at the Faculty of Medicine, ULB-Erasme. MM is supported by the FNRS-Belgium and the Fonds Erasme.

\section{Compliance with ethical standards}

Conflicts of interest $\mathrm{MM}$ has interacted with Guerbet (France) to design experimental studies on the mechanisms of deposits of gadolinium chelates in the rodent brain.

Ethical standards This retrospective study was approved by the Ethical Committee of the ULB-Erasme Hospital.

\section{References}

1. Agris J, Pietsch H, Balzer T (2016) What evidence is there that gadobutrol causes increasing signal intensity within the dentate nucleus and globus pallidus on unenhanced T1W MRI in patients with RRMS? Eur Radiol 26:816-817. doi:10.1007/ s00330-015-4019-2

2. Bauer K, Lathrum A, Raslan O, Kelly PV, Zhou Y, Hewing D, Botkin C, Turner JA, Osman M (2017) Do gadolinium-based contrast agents affect (18)F-FDG PET/CT uptake in the dentate nucleus and the globus pallidus? A pilot study. J Nucl Med Technol 45:302-305. doi:10.2967/jnmt.116.180844

3. Burke LMB, Ramalho M, AlObaidy M, Chang E, Jay M, Semelka RC (2016) Self-reported gadolinium toxicity: a survey of patients with chronic symptoms. Magn Reson Imaging 34:1078-1080. doi:10.1016/j.mri.2016.05.005

4. Cao Y, Huang DQ, Shih G, Prince MR (2016) Signal change in the dentate nucleus on T1-weighted MR images after multiple administrations of gadopentetate dimeglumine versus gadobutrol. AJR Am J Roentgenol 206:414-419. doi:10.2214/AJR.15.15327

5. Cockcroft DW, Gault MH (1976) Prediction of creatinine clearance from serum creatinine. Nephron 16:31-41

6. Grobner T, Prischl FC (2007) Gadolinium and nephrogenic systemic fibrosis. Kidney Int 72:260-264. doi:10.1038/sj.ki.5002338

7. Hao D, Ai T, Goerner F, Hu X, Runge VM, Tweedle M (2012) MRI contrast agents: basic chemistry and safety. J Magn Reson Imaging JMRI 36:1060-1071. doi:10.1002/jmri.23725

8. Higashi M, Irioka T, Matsumoto T, Mizusawa H (2013) Metronidazole-induced encephalopathy. Intern Med Tokyo Jpn 52:843-844

9. High WA, Ayers RA, Chandler J, Zito G, Cowper SE (2007) Gadolinium is detectable within the tissue of patients with nephrogenic systemic fibrosis. J Am Acad Dermatol 56:21-26. doi:10.1016/j.jaad.2006.10.047

10. Hu HH, Pokorney A, Towbin RB, Miller JH (2016) Increased signal intensities in the dentate nucleus and globus pallidus on unenhanced T1-weighted images: evidence in children undergoing multiple gadolinium MRI exams. Pediatr Radiol 46:1590-1598. doi:10.1007/s00247-016-3646-3

11. Hui FK, Mullins M (2009) Persistence of gadolinium contrast enhancement in CSF: a possible harbinger of gadolinium neurotoxicity? AJNR Am J Neuroradiol 30:E1. doi:10.3174/ajnr.A1205 
12. Kanda T, Fukusato T, Matsuda M, Toyoda K, Oba H, Kotoku J, Haruyama T, Kitajima K, Furui S (2015) Gadolinium-based contrast agent accumulates in the brain even in subjects without severe renal dysfunction: evaluation of autopsy brain specimens with inductively coupled plasma mass spectroscopy. Radiology 276:228-232

13. Kanda T, Ishii K, Kawaguchi H, Kitajima K, Takenaka D (2014) High signal intensity in the dentate nucleus and globus pallidus on unenhanced T1-weighted MR images: relationship with increasing cumulative dose of a gadolinium-based contrast material. Radiology 270:834-841. doi:10.1148/radiol.13131669

14. Kanda T, Osawa M, Oba H, Toyoda K, Kotoku JI, Haruyama T, Takeshita K, Furui S (2015) High signal intensity in dentate nucleus on unenhanced T1-weighted MR images: association with linear versus macrocyclic gadolinium chelate administration. Radiology 275:803-809. doi:10.1148/radiol.14140364

15. Lohrke J, Frisk A-L, Frenzel T, Schöckel L, Rosenbruch M, Jost G, Lenhard DC, Sieber MA, Nischwitz V, Küppers A, Pietsch H (2017) Histology and gadolinium distribution in the rodent brain after the administration of cumulative high doses of linear and macrocyclic gadolinium-based contrast agents. Invest Radiol. doi:10.1097/RLI.0000000000000344

16. Manto M (2012) Toxic agents causing cerebellar ataxias. Handb Clin Neurol 103:201-213. doi:10.1016/ B978-0-444-51892-7.00012-7

17. Manto M, Mariën P (2015) Schmahmann's syndrome-identification of the third cornerstone of clinical ataxiology. Cerebellum Ataxias 2:2. doi:10.1186/s40673-015-0023-1

18. Maramattom BV, Manno EM, Wijdicks EFM, Lindell EP (2005) Gadolinium encephalopathy in a patient with renal failure. Neurology 64:1276-1278. doi:10.1212/01.WNL.0000156805.45547.6E

19. McDonald RJ, McDonald JS, Kallmes DF, Jentoft ME, Murray DL, Thielen KR, Williamson EE, Eckel LJ (2015) Intracranial gadolinium deposition after contrast-enhanced MR imaging. Radiology 275:772-782. doi:10.1148/radiol.15150025

20. Murata N, Gonzalez-Cuyar LF, Murata K, Fligner C, Dills R, Hippe D, Maravilla KR (2016) Macrocyclic and other non-group 1 gadolinium contrast agents deposit low levels of gadolinium in brain and bone tissue: preliminary results from 9 patients with normal renal function. Invest Radiol 51:447-453. doi:10.1097/ RLI.0000000000000252

21. Radbruch A, Haase R, Kieslich PJ, Weberling LD, Kickingereder P, Wick W, Schlemmer H-P, Bendszus M (2017) No signal intensity increase in the dentate nucleus on unenhanced T1-weighted MR images after more than 20 serial injections of macrocyclic gadolinium-based contrast agents. Radiology 282:699-707. doi:10.1148/radiol.2016162241

22. Radbruch A, Weberling LD, Kieslich PJ, Eidel O, Burth S, Kickingereder P, Heiland S, Wick W, Schlemmer H-P, Bendszus M (2015) Gadolinium retention in the dentate nucleus and globus pallidus is dependent on the class of contrast agent. Radiology 275:783-791. doi:10.1148/radiol.2015150337

23. Ramalho J, Ramalho M, Jay M, Burke LM, Semelka RC (2016) Gadolinium toxicity and treatment. Magn Reson Imaging 34:1394-1398. doi:10.1016/j.mri.2016.09.005

24. Ramalho J, Semelka RC, AlObaidy M, Ramalho M, Nunes RH, Castillo M (2016) Signal intensity change on unenhanced T1-weighted images in dentate nucleus following gadobenate dimeglumine in patients with and without previous multiple administrations of gadodiamide. Eur Radiol 26:4080-4088. doi:10.1007/s00330-016-4269-7

25. Ray DE, Holton JL, Nolan CC, Cavanagh JB, Harpur ES (1998) Neurotoxic potential of gadodiamide after injection into the lateral cerebral ventricle of rats. AJNR Am J Neuroradiol 19:1455-1462

26. Robert P, Lehericy S, Grand S, Violas X, Fretellier N, Idée J-M, Ballet S, Corot C (2015) T1-weighted hypersignal in the deep cerebellar nuclei after repeated administrations of gadoliniumbased contrast agents in healthy rats: difference between linear and macrocyclic agents. Invest Radiol 50:473-480. doi:10.1097/ RLI.0000000000000181

27. Roberts DR, Welsh CA, LeBel DP, Davis WC (2017) Distribution map of gadolinium deposition within the cerebellum following GBCA administration. Neurology 88:1206-1208. doi:10.1212/ WNL.0000000000003735

28. Runge VM (2000) Safety of approved MR contrast media for intravenous injection. J Magn Reson Imaging JMRI 12:205-213

29. Schwartz GJ, Gauthier B (1985) A simple estimate of glomerular filtration rate in adolescent boys. J Pediatr 106:522-526

30. Semelka RC, Ramalho J, Vakharia A, AlObaidy M, Burke LM, Jay M, Ramalho M (2016) Gadolinium deposition disease: initial description of a disease that has been around for a while. Magn Reson Imaging 34:1383-1390. doi:10.1016/j.mri.2016.07.016

31. Stojanov DA, Aracki-Trenkic A, Vojinovic S, Benedeto-Stojanov D, Ljubisavljevic S (2016) Increasing signal intensity within the dentate nucleus and globus pallidus on unenhanced T1W magnetic resonance images in patients with relapsing-remitting multiple sclerosis: correlation with cumulative dose of a macrocyclic gadolinium-based contrast agent, gadobutrol. Eur Radiol 26:807-815. doi:10.1007/s00330-015-3879-9

32. Woodruff BK, Wijdicks EFM, Marshall WF (2002) Reversible metronidazole-induced lesions of the cerebellar dentate nuclei. N Engl J Med 346:68-69. doi:10.1056/NEJM200201033460117

33. Zhou Z, Lu Z-R (2013) Gadolinium-based contrast agents for MR cancer imaging. Wiley Interdiscip Rev Nanomed Nanobiotechnol 5:1-18. doi:10.1002/wnan.1198 